\title{
STUdy ANALYsis ON Tracking MULTIPLE OBJECTS IN PRESENCE OF INTER OCCLUSION IN UNMANNED LC
}

\author{
A.RISVIN ${ }^{1}$, S.V.ANANDHI ${ }^{2}$, P.SAHANA ${ }^{3}$ \\ ${ }^{1,3}$ Department of Computer Science and Engineering, Dr.Sivanthi Aditanar College of \\ Engineering, Tiruchendur. \\ ${ }^{2}$ Department of Computer Science and Engineering, Dr.Sivanthi Aditanar College of \\ Engineering, Tiruchendur.
}

\begin{abstract}
The object tracking algorithm is used to tracking multiple objects in a video streams. This paper provides Mutual tracking algorithm which improve the estimation inaccuracy and the robustness of clutter environment when it uses Kalman Filter. using this algorithm to avoid the problem of id switch in continuing occlusions. First the algorithms apply the collision avoidance model to separate the nearby trajectories. Suppose occurring inter occlusion the aggregate model splits into several parts and use only visible parts perform tracking. The algorithm reinitializes the particles when the tracker is fully occluded. The experimental results using unmanned level crossing $(L C)$ exhibit the feasibility of our proposal. In addition, comparison with Kalman filter trackers has also been performed.
\end{abstract}

\section{KEYWORD}

Mutual tracking, collision avoidance model, inter occlusion

\section{INTRODUCTION}

\subsection{RELATED WORK}

Now a day's automatic tracking becomes progressively more important in low -cost and smallscale image sensors which are used to deploy surveillance system across unmanned Lc which renders any manual check of videos a very extensive task[1]. The tracking failure causes by occlusion, handling of background clutter environment and illumination changes. The search mechanisms to locate the target can be classified into gradient descent method where some prespecified motion models as Kalman filter. The main role in estimation process in both cases is to match the appearance model of the region predicted by spatiotemporal model. Using Kalman filter [3]in multiple object tracking does not predicting the overlap condition and also is to estimate the state of a linear system where the state is implicit to the distributed by a Gaussian method. Such trackers does not need a beyond a initial bounding box(pretraining) and frequently yield locally optimal solutions in clear scenario of overlapping, shape deformations and lighting conditions. 
Nevertheless such filters [4] are also vulnerable to local minima which can cause divergence. In addition that the appearance model of the target which might also change over time due to changing in light condition. To overcome this problem using mutual tracking algorithm for tracking multiple objects which is the simplest way is likely to use multiple independent tracker (M.I.T), where each target is related to an independent tracker not considering of the state of the other targets. Such approach has been working with some success to follow hockey players and tracking multiple people in unmanned LC in real time[5].

\section{Mutual ObJect Tracking}

The mutual object tracker to track multiple objects when frequent interaction between such objects occlusion problem and also the problem of identity switch. To overcome this problem using mechanism for monitoring all pairs of target estimation [9]. Initially in mutual object tracking measure the distance between any pair wise target estimates is constantly monitored. In this algorithm four cases can be reported.

$>$ The distances are large then use multiple independent tracking which indicating the absence of occlusion and target identity switch.

$>$ If distance is smaller than predefined threshold but without causing of two bounding box then use adaptive collision avoidance model will be applied

$>$ Overlapping between the two target estimation which exists then a partial occlusion based reasoning will be applied and distinguish the occluded target from the non occluded target.

$>$ If the distance indicates a full occlusion which means one bounding box is fully included into the other one then the full occlusion reasoning is activated, where one waits for reappearance of the target. In figure 1 illustrates the mutual object tracking as below

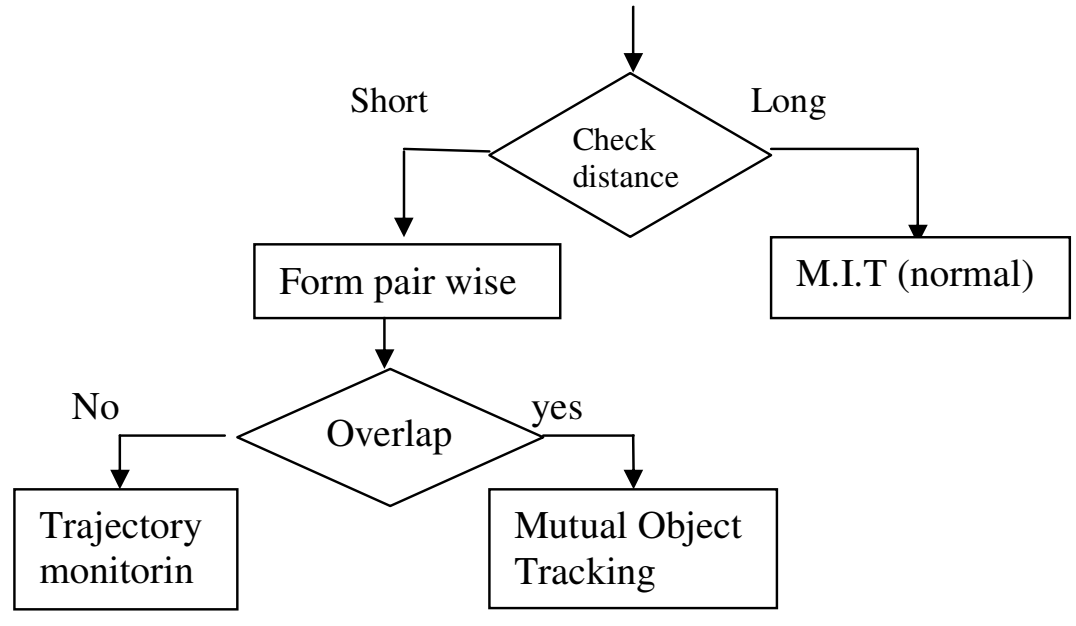

Figure 1. Flow chart of mutual tracking algorithm

\subsection{Adaptive Collision Avoidance Model}

The adaptive collision avoidance model estimates the distance of two targets is less than some predefined threshold and the regions are non overlapping, then compute the weights of the particles in the next frame will be sophisticated by consider both the distance to the other target and dissimilarity of the appearance of the two targets[6]. 
Let as consider the two targets $\mathrm{X} 1$ and $\mathrm{X} 2$ objects in the video that one wants to track, with particles $\left\{\mathrm{L}^{(\mathrm{i})}{ }_{\mathrm{X} 1(\mathrm{k})}, \mathrm{w}^{(\mathrm{i})}{ }_{\mathrm{X} 1(\mathrm{k})}\right.$. The weight of the current $\mathrm{w}^{(\mathrm{j})}{ }_{\mathrm{X} 1}$ of the jth particle will be calculated as

$$
\mathrm{w}^{,{ }^{(j)}}{ }_{\mathrm{X} 1}=\mathrm{w}^{(\mathrm{j})}{ }_{\mathrm{X} 1} \exp \left(\lambda_{\mathrm{X} 1 \mathrm{X} 2} \cdot \mathrm{d}\left(\mathrm{R}^{\mathrm{X} 1}{ }_{\mathrm{j}}, \mathrm{R}^{\mathrm{X} 2}\right)\right)
$$

Where $\mathrm{R}^{\mathrm{X} 1}{ }_{\mathrm{j}}$ and $\mathrm{R}^{\mathrm{X} 2}$ are bounding box regions associated to jth particle of target $\mathrm{X} 1$ and global estimate of target $\mathrm{X} 2$ respectively. $\mathrm{d}\left(\mathrm{R}_{\mathrm{j} 1}^{\mathrm{X}}, \mathrm{R}^{\mathrm{X} 2}\right)$ is the distance between the two bounding box. Similar responding applies to particle $\mathrm{j}$ of target $\mathrm{X} 2$, where the counterpart of (2.1) is

$$
\mathrm{w}^{\prime}{ }^{(\mathrm{j})}{ }_{\mathrm{x} 2}=\mathrm{w}^{(\mathrm{j})}{ }_{\mathrm{x} 2} \exp \left(\lambda_{\mathrm{x} 1 \mathrm{X} 2} \cdot \mathrm{d}\left(\mathrm{R}^{\mathrm{X} 2}{ }_{\mathrm{j}}, \mathrm{R}^{\mathrm{X} 1}\right)\right)
$$

Where

$\lambda_{\mathrm{X} 1 \mathrm{X} 2}-$ similarity of the appearance models associated to the two targets.

\subsection{Hierarchical Tracking Priority}

In the case partial occlusion the minor overlapping this cannot be excluded that the actual shapes of two targets are completely identified without any uncertainty. If the two targets have similar appearance model then use alternative reasoning will be required.

$$
\mathrm{B}\left(\mathrm{A}^{\mathrm{X} 1}, \mathrm{~A}^{\mathrm{X} 2}\right) \geq \mathrm{T}_{\mathrm{a}}
$$

The equation (2.3) indicates that the appearance models between the two target is deemed To be similar and their similarity values in the sense of Bhattacharya coefficient is larger than some predefined threshold $\mathrm{T}_{\mathrm{a}}$.

\subsubsection{DifFERENT TARget APPEARANCE MODELS}

Once the occlusion is conformed between the two bounding box regions then propose a two step mechanism to conform the existence of the occlusion [8]. First compute the likelihood $\mathrm{w}_{\mathrm{x} 1}$ and $\mathrm{w}_{\mathrm{x} 2}$ using the appearance model $\mathrm{A}^{\mathrm{x} 1}$ and $\mathrm{A}^{\mathrm{x} 2}$ around the global estimated bounding box of each target. Second, to differentiate between the cases where the discrepancy of the two likelihood is due to a wrong estimation.

Let us assume without loss of generality that,

$$
\mathrm{w}_{\mathrm{x} 1}<\mathrm{w}_{\mathrm{x} 2}
$$

Then calculate the positive contribution of the appearance model of another target X2 over the X1 reference model, which can be calculated by

$$
\begin{aligned}
& \mathrm{h}_{1}^{\mathrm{X} 1}=\sum \quad\left(\mathrm{A}^{\mathrm{x} 2(\mathrm{i})}-\mathrm{A}^{\mathrm{refx} 1}(\mathrm{i})\right) \\
& \mathrm{i},\left[\mathrm{A}{ }^{\mathrm{X} 2}{ }_{(\mathrm{i})-\mathrm{A}^{\mathrm{refX} 1}}\right. \text { (i)]>0}
\end{aligned}
$$

Next repeat the same reasoning with enlarged region of target $\mathrm{x} 1$ and focusing on background region, yielding

$$
\mathrm{H}_{2}^{\mathrm{X} 1}=\sum_{\mathrm{i},\left[\mathrm{A}^{\mathrm{xb} 1}(\mathrm{i})-\mathrm{A}^{\mathrm{refX} 1}\right.}^{(\mathrm{i})]>0}
$$


where, $\mathrm{A}_{\mathrm{b} 1}^{\mathrm{X}}$ indicates the histogram associated to background region of the enlarged bounding box pertaining to target $\mathrm{x} 1$.Comparing (2.5) and (2.6)

$$
\mathrm{h}^{\mathrm{x} 1}{ }_{1}>\mathrm{h}^{\mathrm{x} 1}{ }_{2}
$$

In this $X_{1}$ is assumed to be occluded by $X_{2}$.otherwise the occlusion cannot be confirmed in the current frame. If the occlusion is confirmed first stop enabling the adaptive collision avoidance model and second to determine the visible and non visible parts in bounding box regions of targets and third to recomputes the weights accordingly.

Target $X_{1}$

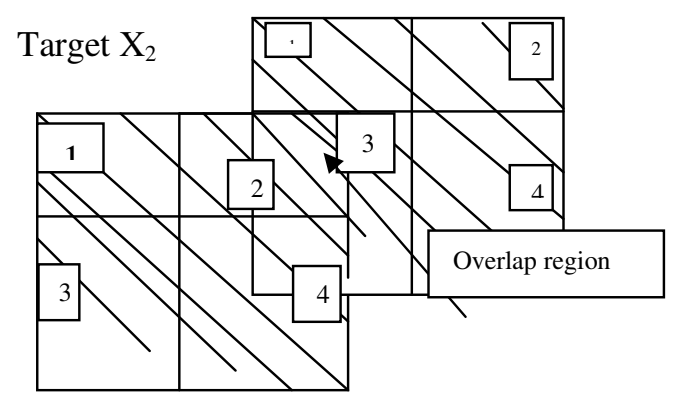

Figure 2: Overlapping of the two target estimates

In figure 2, a simple case of target overlapping which conforms a partial occlusion. This approach which create a subdivision of each bounding box region to create for visible and non visible parts. The initial outcome of such occlusion reasoning is twofold.

1)In the case of figure 2,ignoring any occlusion scenario the visible part of target $X 2$ corresponds to partitions $1,, 4$,while that of target X1corresponds to partition 2 and 4.This is called as visual partition model .If the occlusion reasoning occluded that only target $\mathrm{X} 2$ is partially occluded ,then the target X1 becomes fully visible, while partition of 1,3, and 4 are the visible parts of target X2

2) The new weight is computed for each visible parts only. More formally each subdivision $\mathrm{j}$ of the bounding box associated to target $\mathrm{X}_{\mathrm{i}}$ Next considering a set $\mathrm{V}$ of subdivision that belongs to visible part, the new weight is computed for the target $X_{i}$ as

With

$$
\mathrm{w}_{\mathrm{v}} \mathrm{X}_{\mathrm{i}}=1 / \mathrm{VI} \sum_{\mathrm{j} € \mathrm{~V}} \mathrm{w}^{(\mathrm{sj})}{ }_{\mathrm{Xi}}
$$

$$
\mathrm{w}^{(\mathrm{sj})}{ }_{\mathrm{Xi}}=\mathrm{e}^{\lambda \mathrm{aB}}\left(\mathrm{A}_{\mathrm{sj}}^{\mathrm{Xi}}, \mathrm{A}^{\mathrm{ref}}{ }_{\mathrm{Xi}}\right)
$$

Where, visible in (2.8)

\subsubsection{SAME TARget APPEARANCE MODElS}

The targets have a same appearance models then the above reasoning cannot be employed to conform of an occlusion. In this method monitor the trajectory of the targets and adjusting the weight of the particles according to the direction of the target movement and also considering the size of the bounding box regions. First determine the direction of movement the target for this use. First monitoring the velocity of the center of the bounding box region within a predefined time window. Let $(\mathrm{Vx}, \mathrm{Vy})$ be the average velocity of a given tracker which is computed from 
previous $\mathrm{m}$ frames then one can use the sign of the largest absolute values between $\mathrm{Vx}$ and $\mathrm{Vy}$ to decide on the direction as it shown in table I.

Table I Direction Map.

\begin{tabular}{|c|c|c|}
\hline $\operatorname{Max}\left(\left|\mathrm{V}_{\mathrm{x}}\right|,\left|\mathrm{V}_{\mathrm{y}}\right|\right)$ & Sign of $\mathrm{V}_{\mathrm{x}}, \mathrm{V}_{\mathrm{y}}$ & Output(Directions) \\
\hline $\mathrm{V}_{\mathrm{x}}$ & + & Right \\
\hline $\mathrm{V}_{\mathrm{x}}$ & - & Left \\
\hline $\mathrm{V}_{\mathrm{y}}$ & + & Down \\
\hline $\mathrm{V}_{\mathrm{y}}$ & - & Up \\
\hline
\end{tabular}

\subsubsection{FULL OCCLUSION BEFORE TARGET RETRACKING}

In case of full occlusion relies on the concept of object performance, which suggests that a fully occluded target will reemerge from its occluder[10].In addition it is typically known that the weight associated to occluding target particles are usually low. The idea [10] is to randomly reinitialize the particles of the occluded target around the occluder, from that the tracker can easily capture the reappeared target immediately after its reappearance in Figure 3.

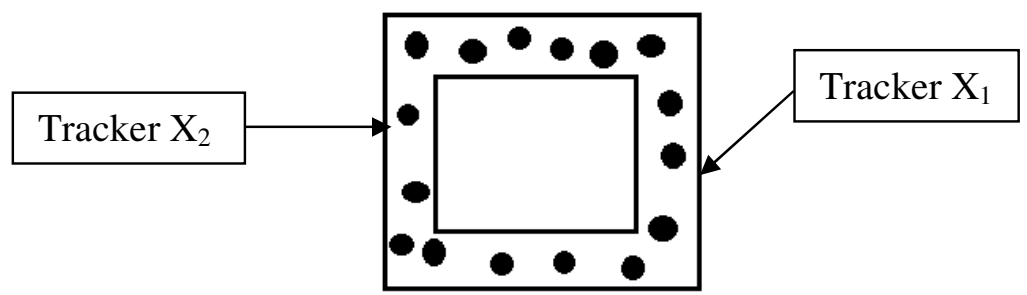

Figure 3: Full occlusion (small circles: reinitialize particles)

The tracker will compare the appearance of newly estimated tracker to the reference model for confirming the reemerge tracker. The threshold value set $T_{r}=0.8$, then the target conform the reappeared target. Otherwise the tracker will perform reinitialize the particles based upon the occluded target position.

\section{EXPERIMENTS AND RESULTS}

In this section, we first test the unmanned Lc video for object identification using Kalman filter. Using Kalman filter which does not predict the object overlapping shown in Figure 4

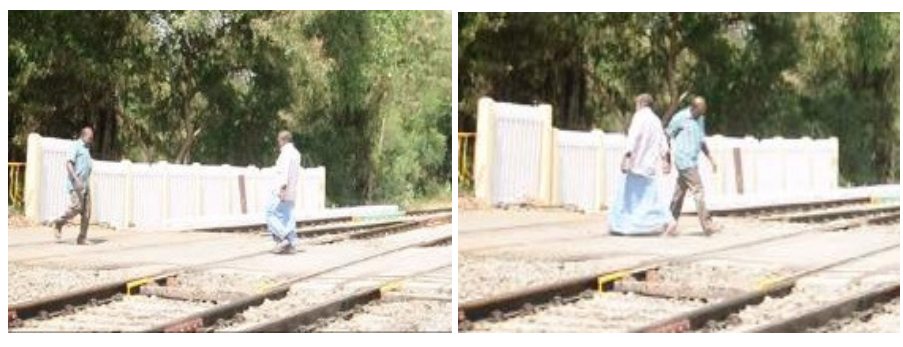

(a) Frame 12

(b) Frame 53 
Advanced Computational Intelligence: An International Journal (ACII), Vol.3, No.3, July 2016

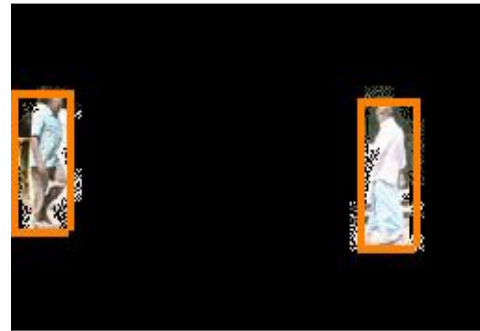

(c) Frame 12

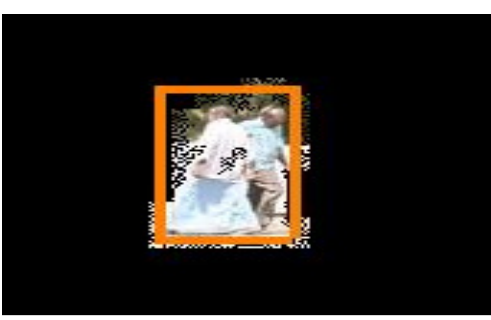

(e)Frame 53

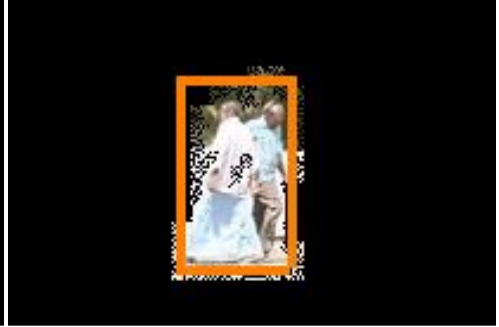

(d)Frame 52

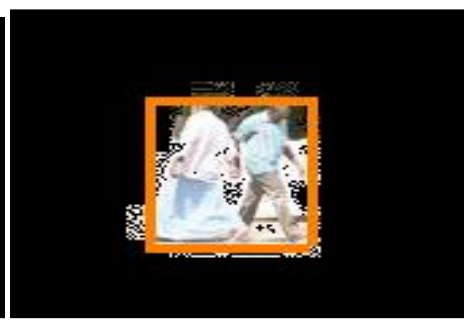

(f) Frame 54

Figure 4: Multiple object tracking performance using Kalman filter.

In this frame 53 the occlusion is occurred but it does not overlapping the id switch problem. This is major drawback of using Kalman filter in multiple object tracking.

To better multiple objects tracking in inter occlusion using mutual tacking algorithm for our experiment. Figure 5 shows the performance of Mutual tracking algorithm on Unmanned Lc.

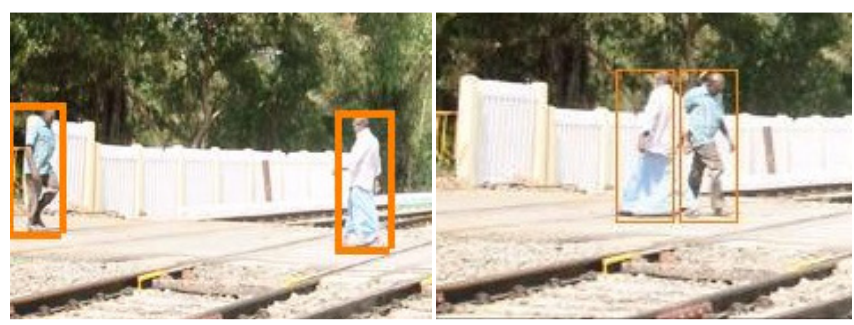

(a)Frame12

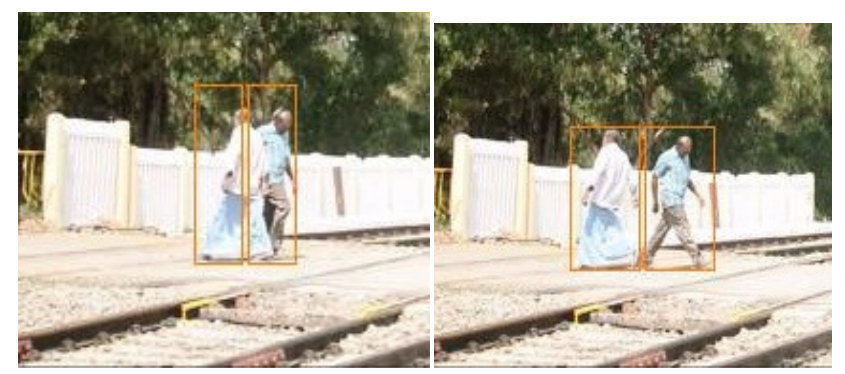

(a)Frame53 (b) Frame52

(c) Frame 54

Figure 5: Multiple object tracking performance using Mutual tracking Algorithm. 
Advanced Computational Intelligence: An International Journal (ACII), Vol.3, No.3, July 2016

\section{CONCLUSION}

This study presents tracking multiple objects using mutual tracking algorithm.First,to deal with arising uncertainty from background clutter and changing illumination of light in background, the contextual information is considered by enlarging the boundary of the estimated target region and comparing with current observation.Second,the particles distribution is considered through the recursive estimation that restricts the effect of outliers on global estimate of the target. Third to track multiple objects, although the intuitive use of multiple independent tracker M.I.T where each tracker is associated to an individual target, cautious is required to avoid the problem of occlusion or identity switch. In this deal with the problem by monitoring the distance between trackers. In case of partial occlusion where each particle is partitioned into equal partition and only the visible parts of the partition are used for tracking. In case of full occlusion, to reinitialize the particles around the occluder to capture the reappearance of target. An experimental result shows comparisons with Kalman filter and mutual tracking algorithm using Unmanned LC video which deals with occlusions, clutter and the illumination change.

\section{REFERENCES}

[1] X. Wang, "Intelligent multi-camera video surveillance: A review,"Pattern Recognit. Lett., vol. 34, no. 1, pp. 3-19, Jan. 2013.

[2] D. Comaniciu, V. Ramesh, and P. Meer, "Real-time tracking of non-rigid objects using mean shift," in Proc. IEEE Conf. CVPR, vol. 2. Jun. 2000,pp. 142-149.

[3] S.-K. Weng, C.-M. Kuo and S.-K. Tu, "Video object tracking using adaptive Kalman filter," J. Vis. Commun. Image Represent. vol. 17, no. 6,pp. 1190-1208, Dec. 2006.

[4] Y. Wu, J. Lim, and M.-H. Yang, “Online object tracking: A benchmark,"in Proc. IEEE CVPR, Jun. 2013, pp. 2411-2418.

[5] K. Nummiaro, E. Koller-Meier, and L. Van Gool, “An adaptive colorbased particle filter," Image Vis. Comput., vol. 21, no. 1, pp. 99-110,Jan. 2003.

[6] V. Papadourakis and A. Argyros, "Multiple objects tracking in the presence of long-term occlusions," Comput. Vis. Image Understand., vol. 114, no. 7, pp. 835-846, Jul.2010.

[7] K. Okuma, A. Taleghani, N. de Freitas, J. J. Little, and D. G. Lowe, "A boosted particle filter: Multitarget detection and tracking," in Proc.ECCV, 2004, pp. 28-39.

[8] W. Du and J. Piater, "Multi-camera people tracking by collaborative particle filters and principal axis-based integration," in Proc. ACCV, 2007, pp. 365-374.

[9] H. Ben Shitrit, J. Berclaz, F. Fleuret, and P. Fua, "Tracking multiple people under global appearance constraints," in Proc. IEEE ICCV, Nov. 2011, pp. 137-144.

[10] V. Papadourakis and A. Argyros, "Multiple objects tracking in the presence of long-term occlusions," Comput. Vis. Image Understand., vol. 114, no. 7, pp. 835-846, Jul. 2010.

[11] S. Pellegrini, A. Ess, K. Schindler, and L. Van Gool, "You'll never walk alone: Modelling social behavior for multi-target tracking," in Proc. IEEE ICCV, Sep./Oct. 2009, pp. 261-268.

[12] S. Hare, A. Saffari, and P. H. S. Torr, "Struck: Structured output tracking with kernels," in Proc. ICCV, Nov. 2011, pp. 263-270.

[13] X. Mei and H. Ling, "Robust visual tracking using _1 minimization, "in Proc. IEEE ICCV, Sep./Oct. 2009, pp. 1436-1443.

[14] J. F. Henriques, R. Caseiro, P. Martins, and J. Batista, "Exploiting the circulant structure of trackingby-detection with kernels," in Proc.ECCV, 2012, pp. 702-715.

[15] F. Pernici and A. Del Bimbo, "Object tracking by oversampling local features," IEEE Trans. Pattern Anal. Mach. Intell., vol. 36, no. 12, pp. 2538-2551, Dec. 2014.

[16] Video Benchmark Data Set. [Online]. Available:

http://cvlab.hanyang.ac.kr/tracker_benchmark/seq/Subway.zip, accessed Feb. 2015. 